\title{
Cardiac tamponade as a rare complication after giant coronary fistula percutaneous closure
}

\author{
Pablo Díez Villanueva*, Fernando Sarnago Cebada, Enrique Gutiérrez Ibañes, Ricardo Sanz-Ruíz, \\ Jaime Elízaga-Corrales, Francisco Fernández-Avilés \\ Cardiology Department, Hospital General Universitario Gregorio Marañón, Madrid, Spain \\ Email: *pablo_diez_villanueva@hotmail.com
}

Received 12 January 2013; revised 8 March 2013; accepted 13 April 2013

Copyright (C) 2013 Pablo Díez Villanueva et al. This is an open access article distributed under the Creative Commons Attribution License, which permits unrestricted use, distribution, and reproduction in any medium, provided the original work is properly cited.

\begin{abstract}
We present the case of an unusual complication after percutaneous closure of a giant coronary artery fistula. A 76-year-old man with previous admissions due to right heart failure and previous history of atrial fibrillation under acenocumarol, was admitted to our hospital for new onset of symptoms, characterized by progressive dyspnoea and peripheral edema. Physical examination revealed signs of congestive heart failure and a continuous murmur loudest along the lower sternal border. X-Ray showed cardiomegaly due to right chambers dilatation. Transthoracic echocardiography showed right chambers pressure and volume overload, with right ventricular enlargement and dysfunction, tricuspid annulus dilatation and severe tricuspid regurgitation. Cardiac catheterization showed significant elevation of right atrial pressure, as well as significant step-up of oxygen saturation in this chamber. Coronary angiography revealed the presence of a large fistula between the circumflex coronary artery (CCA) and coronary sinus (CS), with severe dilation of the CCA (maximum diameter 20 mm). An Amplatzer ${ }^{\circledR}$ PDA was implanted in a distal elbow of the fistula with initailly good results. Anticoagulant therapy was then reinitiated, and a few days later, the patient developed clinical worsening of heart failure and dyspnoea. Echocardiogram showed significant pericardial effusion. Pleuropericardial window was then made draining a $500 \mathrm{~cm}^{3}$ of bloody pericardial effusion. The postoperative outcome was excellent, with symptomatic relief and no signs of heart failure.
\end{abstract}

Keywords: Giant Coronary Fistula; Circumflex Coronary Artery; Coronary Sinus; Percutaneous Transcatheter Closure; Pericardial Effusion; Cardiac

${ }^{*}$ Corresponding author.
Tamponade

\section{INTRODUCTION}

Coronary artery fistulas (CFA) are rare, usually congenital entities, resulting in abnormal communications between a coronary artery and, usually, any low-pressure structure, entailing a left-to-right shunt bypassing the capillary bed. Such fistulas most often arise from the right coronary artery, while the circumflex coronary artery is rarely involved. There is a wide range of symptoms; most patients may be asymptomatic, while others may present with dyspnea, congestive heart failure or angina.

Management of asymptomatic patients with small coronary artery fistula remains controversial, as the natural history of coronary artery fistulas is variable. On the other hand, surgical or percutaneous closure is indicated in case of symptomatic or significant shunt. Bloody pericardial effusion as main complication after a percutaneous intervention had never been described as a post-percutaneous procedure complication.

\section{CASE DESCRIPTION}

A 76-year-old male, with previous admissions for right heart failure, was admitted to our hospital for new onset of symptoms. He also had previous history of atrial fibrillation, and anticoagulant therapy with acenocoumarol. Physical examination revealed signs of congestive heart failure and a continuous murmur loudest along the lower sternal border. X-Ray showed cardiomegaly due to right chambers dilatation.

Echocardiogram showed volume overload of a severely dilated and hypocontractile right ventricle, and severe tricuspid insufficiency due to annulus dilatation. Pulmonary artery systolic pressure was estimated at 67 $\mathrm{mmHg}$. A vascular structure in the atrioventricular groove 
compatible with dilated coronary sinus was also described.

Cardiac catheterization evidenced significant elevation of right atrium pressure $(27 \mathrm{mmHg})$ and oximetry data revealed a significant step-up of oxygen saturation in the right atrium (average of both the superior and inferior vena cava $60 \%$, and right ventricle $71 \%$ ). Cardiac output was $7.7 \mathrm{l} / \mathrm{min}$ and Qp/Qs was 2.5. There was pulmonary hypertension (57/36/26 mmHg) and pulmonary capillary wedge pressure was $27 \mathrm{mmHg}$. Coronary angiography evidenced a large fistula between the circumflex coronary artery (CCA) and the coronary sinus (CS), arising from the distal part of the artery, which was severely dilated (maximum diameter $20 \mathrm{~mm}$ ). The right coronary artery was normal.

An Amplatzer PDA ${ }^{\circledR}$ (patent ductus arteriosus device, $16 / 14 \mathrm{~mm}$ ), slightly oversized to try to stop the high flow shunt, was implanted by forming an arteriovenous wire loop, in a distal elbow of the fistula with minimal residual flow (Figure 1 shows the angiography preprocedure, the arteriovenous wire loop, and the angiography after deploying the device. Another projection of the final result is shown in Figure 2). Anticoagulation therapy was reinitiated with low molecular weight heparin (0.75 $\mathrm{mg} / \mathrm{mg}$ twice a day, adjusted for age and renal function) the day after the procedure, and oral anticoagulant therapy was initiated two days later.

Five days after the percutaneous closure, the patient

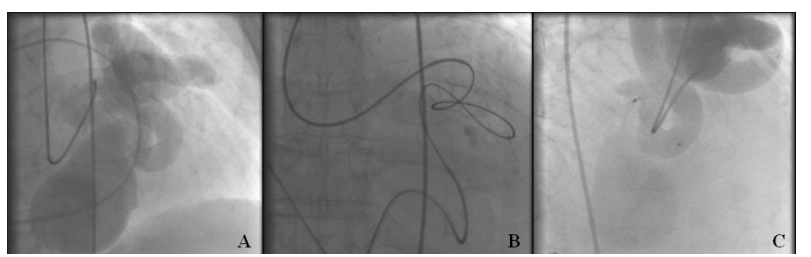

Figure 1. Initial angiography (A); Arteriovenous loop (B); Final angiography $(\mathrm{C})$.

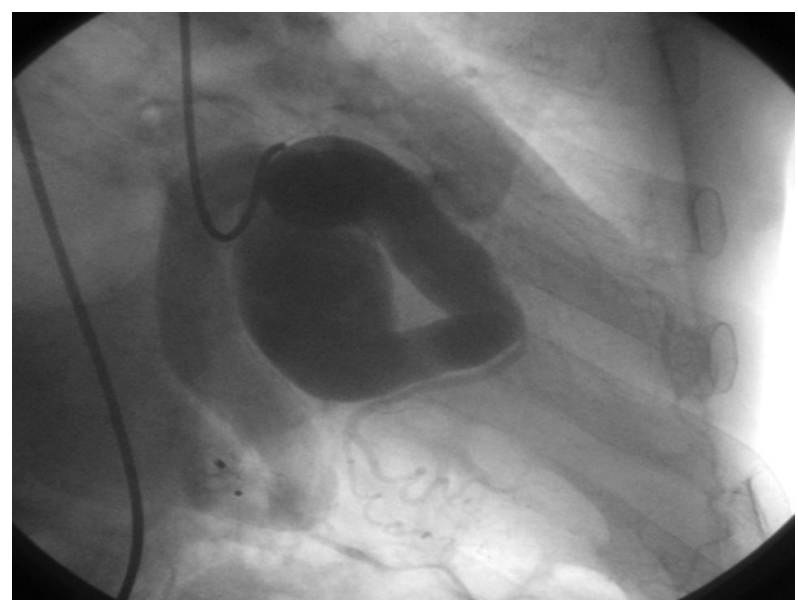

Figure 2. The Amplatzer PDA ${ }^{\circledR}$ device was implanted in a distal elbow of the fistula with minimal residual flow. developed clinical worsening of heart failure and dyspnoea. Echocardiogram showed significant pericardial effusion, predominantly lateral and posterior (Figure 3). International Normalized Ratio, INR, was 2.5. After administering vitamin $\mathrm{K}$ and correct abnormal coagulation, pleuropericardial window was made through left anterolateral thoracotomy, draining a significant amount (500 $\mathrm{cm}^{3}$ ) of bloody pericardial effusion. After that, the postoperative outcome was excellent, with symptomatic relief and no signs of heart failure.

One year later, the patient had no limitation for dyspnea, with great improvement in his physical activity. Previous murmur was absent. Cardiac catheterization demonstrated minimal residual flow through the device, no saturation step-up along the right chambers and Qp/Qs was 1.1.

Cardiac MRI showed the device correctly implanted at the distal portion of the CCA partially thrombosed. Also, right ventricle was not dilated, with normal function, and tricuspid regurgitation was only moderate.

\section{DISCUSSION}

Coronary artery fistulas are rare, usually congenital entities, recognized in approximately $0.2 \%$ of routine coronary angiograms. These malformations are abnormal communications between a coronary artery and any cardiac cavity, great vessel or other low-pressure structure, which gives rise to a left-to-right shunt bypassing the capillary bed [1]. Much more uncommon, CFA communicate with a left heart chamber. When acquired, aetiologies such as coronary atherosclerosis, Takayasu arteritis, and trauma should be considered.

These fistulas most often arise from the right coronary artery, up to $55 \%$ of patients, the left coronary artery in $35 \%$ of patients, and both coronary arteries in $10 \%$ of patients. The circumflex coronary artery is rarely in-

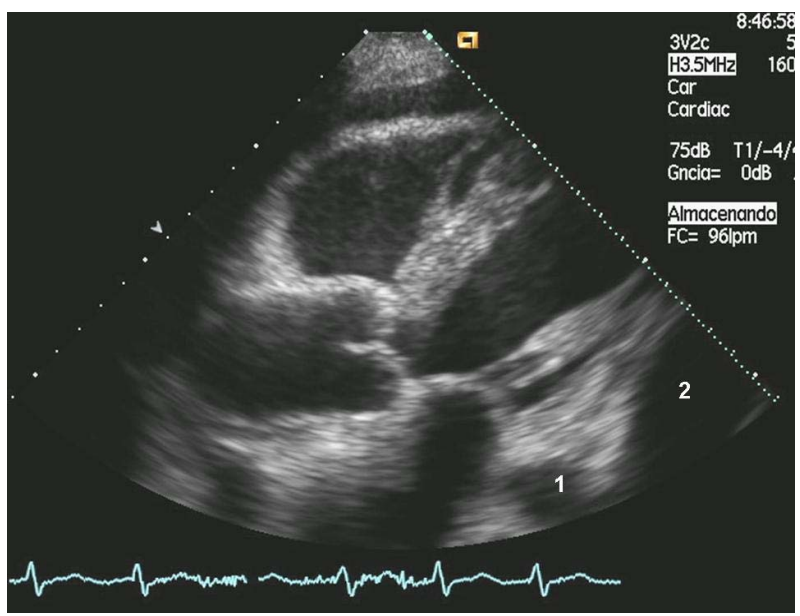

Figure 3. Dilation of the coronary sinus (1); Severe pericardial effusion (2). 
volved [2].

Most patients have no symptoms. Clinical presentation depends on the severity of the shunt and complications tend to increase with age. Dyspnea, congestive heart failure and angina occur in up to $20 \%$ of patients. Other complications, such as arrhythmias and sudden death, myocardial infarction, or endocarditis are less common.

Particularly uncommon, fistulae can also be associated with coronary aneurysms. In such aneurysmal formations, mainly if large and sacular, surgery might be considered, due to rupture risk [3], although good results have been reported with covered stent deployment [4].

The management of asymptomatic patients with small coronary artery fistula $(\mathrm{Qp} / \mathrm{Q} s<1.5)$ remains controversial, as the natural history of coronary artery fistulas is variable. Both surgical ligation and percutaneous occlusion should be considered for patients with complications, as it has been well demonstrated that these techniques are safe and effective, and can be performed with a very low risk. Therefore, these techniques are indicated in case of big CAF, Qp/Qs > 1.5 or symptomatic.

Bloody pericardial effusion as main complication after the percutaneous intervention, which is uncommon (despite there are some cases reported in the literature of spontaneous rupture and pericardial tamponade [5]), had never been described as a post-percutaneous procedure complication. After implantation of septal amplatzer occluders in patients with patent foramen ovale, erosions have been reported to occur at the roof of the atrium or at the atrial junction with the aorta thus causing hemopericardium or aortic fistula. There is still controversy about the risk factors but many cardiac interventionalists agree that device motion relative to the heart causes erosion, and on that basis it has been recommended to oversize devices and aortic straddling in cases with deficient aortic rim [6]. However, despite that might have been one of the mechanisms suggested in our case, such finding was not confirmed by any imaging technique or during surgical pericardial window. Even more, pericardial effusion could just be related to some kind of perforation due to handling of stiff guidewires or catheters during the procedure. The earliness of the presentation and the good performance with only draining the effusion might suggest the second mechanism. In such case, anticoagulant therapy might have played a role in the setting of a deferred and slow development of pericardial effusion, ensuing pericardial tamponade.

Regarding the patient of our case, it is noteworthy his advanced age, as well as the fact that the fistula was located within the circumflex artery, draining into the venous circulation, in the coronary sinus. Interestingly, older age is known to be a clinical predictor associated with adverse outcomes. Moreover, fistula origin and drainage site are important angiographic features, regarding the fact that both distal origin of the fistula in the artery as well as drainage into the coronary sinus have demonstrated to be predictive of long-term complications (coronary thrombosis, myocardial infarction, and cardiomyopathy) in patients with CAF $[7,8]$. Some authors recommend chronic anticoagulant therapy in such cases. Atrial fibrillation was another indication for such therapy in our patient. Our case enhances the lack of available data and the difficulty of deciding the correct antithrombotic/anticoagulant therapy in these patients. Our patient, however, remains clinically stable in subsequent revisions even under chronic anticoagulant therapy.

\section{REFERENCES}

[1] Gowda, R.M., Vasavada, B.C. and Khan, I.A. (2006) Coronary artery fistulas: Clinical and therapeutic considerations. International Journal of Cardiology, 107, 7-10. doi:10.1016/j.ijcard.2005.01.067

[2] Katoh, T., Zempo, N., Minami, Y., et al. (1999) Coronary arteriovenous fistulas with giant aneurysm: Two case reports. Cardiovascular Surgery, 7, 470-472. doi:10.1016/S0967-2109(98)00102-1

[3] Hajj-Chahine, J., Haddad F., El-Rassi, I., et al. (2009) Surgical management of a circumflex aneurysm with fistula to the coronary sinus. European Journal of Cardio-Thoracic Surgery, 35, 1086-1088. doi:10.1016/j.ejcts.2009.02.039

[4] Ghaffari, S., Akbarzadeh, F. and Pourafkari, L. (2011) Aneurysmal coronary arteriovenous fistula closing with covered stent deployment: Case report and review of literature. Cardiology Journal, 18, 556-559. doi:10.5603/CJ.2011.0013

[5] Gamma, R., Seiler, J., Moschovitis, G., et al. (2006) Giant coronary artery fistula complicated by cardiac tamponade. International Journal of Cardiology, 107, 413414. doi:10.1016/j.ijcard.2005.01.065

[6] El-Said, H.G. and Moore, J.W. (2009) Erosion by the Amplatzer septal occluder: Experienced operator opinions at odds with manufacturer recommendations? Catheterization \& Cardiovascular Interventions, 73, 925-930. doi:10.1002/ccd.21931

[7] Valente, A.M., Lock, J.E., Gauvreau, K., et al. (2010) Predictors of long-term adverse outcomes in patients with congenital coronary artery fistulae. Circulation: Cardiovascular Interventions, 3, 134-139. doi:10.1161/CIRCINTERVENTIONS.109.883884

[8] Gowda, S.T., Latson, L.A., Kutty, S., et al. (2011) Intermediate to long-term outcome following congenital coronary artery fistulae closure with focus on thrombus formation. American Journal of Cardiology, 107, 302-308. doi:10.1016/j.amjcard.2010.09.018 